Louisiana State University

LSU Digital Commons

$5-13-2002$

\title{
Time-frequency characterization of femtosecond extreme ultraviolet pulses
}

J. Norin

Lunds Tekniska Högskola

J. Mauritsson

Lunds Tekniska Högskola

A. Johansson

Lunds Tekniska Högskola

M. K. Raarup

Aarhus Universitet

S. Buil

Lunds Tekniska Högskola

See next page for additional authors

Follow this and additional works at: https://digitalcommons.Isu.edu/physics_astronomy_pubs

\section{Recommended Citation}

Norin, J., Mauritsson, J., Johansson, A., Raarup, M., Buil, S., Persson, A., Dühr, O., Gaarde, M., Schafer, K., Schafer, K., Keller, U., Keller, U., Wahlström, C., \& L'Huillier, A. (2002). Time-frequency characterization of femtosecond extreme ultraviolet pulses. Physical Review Letters, 88 (19), 1939011-1939014.

https://doi.org/10.1103/PhysRevLett.88.193901

This Article is brought to you for free and open access by the Department of Physics \& Astronomy at LSU Digital Commons. It has been accepted for inclusion in Faculty Publications by an authorized administrator of LSU Digital Commons. For more information, please contact ir@lsu.edu. 


\section{Authors}

J. Norin, J. Mauritsson, A. Johansson, M. K. Raarup, S. Buil, A. Persson, O. Dühr, M. B. Gaarde, K. J. Schafer, K. J. Schafer, U. Keller, U. Keller, C. G. Wahlström, and A. L'Huillier 


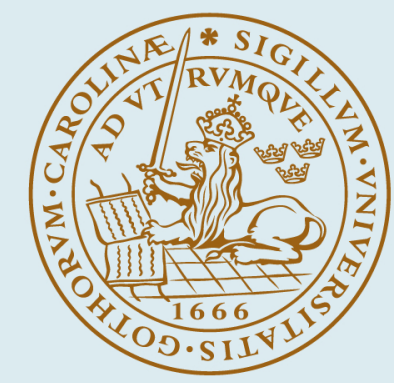

\section{LUND UNIVERSITY}

Time-frequency characterization of femtosecond extreme ultraviolet pulses.

Norin, Johan; Mauritsson, Johan; Johansson, Ann; Raarup, M K; Buil, S; Persson, Anders; Dühr, O; Gaarde, Mette; Schafer, Kenneth; Keller, U; Wahlström, Claes-Göran; L'Huillier, Anne

Published in:

Physical Review Letters

DOI:

10.1103/PhysRevLett.88.193901

2002

Link to publication

Citation for published version (APA):

Norin, J., Mauritsson, J., Johansson, A., Raarup, M. K., Buil, S., Persson, A., Dühr, O., Gaarde, M., Schafer, K., Keller, U., Wahlström, C-G., \& L'Huillier, A. (2002). Time-frequency characterization of femtosecond extreme ultraviolet pulses. Physical Review Letters, 88(19), $193901-193901$.

https://doi.org/10.1103/PhysRevLett.88.193901

Total number of authors:

12

\footnotetext{
General rights

Unless other specific re-use rights are stated the following general rights apply:

Copyright and moral rights for the publications made accessible in the public portal are retained by the authors and/or other copyright owners and it is a condition of accessing publications that users recognise and abide by the legal requirements associated with these rights.

- Users may download and print one copy of any publication from the public portal for the purpose of private study or research.

- You may not further distribute the material or use it for any profit-making activity or commercial gain

- You may freely distribute the URL identifying the publication in the public portal
}

Read more about Creative commons licenses: https://creativecommons.org/licenses/

Take down policy

If you believe that this document breaches copyright please contact us providing details, and we will remove access to the work immediately and investigate your claim. 


\title{
Time-Frequency Characterization of Femtosecond Extreme Ultraviolet Pulses
}

\author{
J. Norin, ${ }^{1}$ J. Mauritsson, ${ }^{1}$ A. Johansson, ${ }^{1}$ M. K. Raarup ${ }^{2, *}$ S. Buil,${ }^{1, \dagger}$ A. Persson, ${ }^{1}$ O. Dühr, ${ }^{1}$ M. B. Gaarde, ${ }^{1}$ \\ K. J. Schafer, ${ }^{1,3}$ U. Keller, ${ }^{1,4}$ C.-G. Wahlström, ${ }^{1}$ and A. L'Huillier ${ }^{1}$ \\ ${ }^{1}$ Department of Physics, Lund Institute of Technology, P.O. Box 118, S-221 00 Lund, Sweden \\ ${ }^{2}$ Institute of Physics and Astronomy, University of Aarhus, DK-8000 Aarhus C, Denmark \\ ${ }^{3}$ Department of Physics and Astronomy, Louisiana State University, Baton Rouge, Louidisns 70803-4001 \\ ${ }^{4}$ Ultrafast Laser Physics Laboratory, Institute of Quantum Electronics, ETH Hönggerberg-HPT, CH-8093 Zürich, Switzerland
}

(Received 14 November 2001; published 25 April 2002)

\begin{abstract}
We present energy-resolved cross-correlation measurements of an extreme ultraviolet (XUV) pulse, generated as the fifth harmonic $(15.5 \mathrm{eV})$ of an intense $80 \mathrm{fs}$ laser pulse centered at $400 \mathrm{~nm}$. Spectrally resolving the cross-correlation signal allows us to characterize the time-dependent frequency of the XUV pulse. We find that the fifth harmonic has a small negative chirp in excess of that predicted by perturbation theory. In addition, by manipulating the chirp of the driving laser we can induce and measure a positive or a negative chirp on the XUV pulse.
\end{abstract}

DOI: $10.1103 /$ PhysRevLett.88.193901

The production of high-order harmonics by an intense laser field is a process that necessarily involves strong-field continuum dynamics, since in the first step of this process the initially bound electron must be excited above the ionization threshold. There is convincing theoretical and experimental evidence that the subsequent continuum dynamics produce a time-dependent frequency modulation on the harmonics [1-6]. The simplest description of this effect is given by the semiclassical model of harmonic generation [7], in which the frequency modulation of the extreme ultraviolet (XUV) radiation originates in the extra phase that the electron wave packet accumulates during its laser-driven acceleration in the continuum [1]. In addition to this single atom effect, phase matching and ionization in the macroscopic medium can also lead to frequency modulations [8-10]. The recent production of attosecond pulse trains [11], which relies on phase locking several high-order harmonics, makes the direct measurement of the time-frequency behavior of the XUV radiation especially timely.

Although the time-frequency characterization of visible and infrared pulses is now standard in many laboratories $[12,13]$ it is nontrivial to extend it to the extreme ultraviolet region, mainly because of the difficulty of inducing nonlinear processes in this region $[14,15]$. Various spectral characterizations, in combination with measurements of the pulse duration $[6,14]$, phase shaping of the fundamental pulse $[3,16]$, or measurements of the temporal coherence properties [2], all suggest that the harmonics are chirped. However, no experimental measurements of the chirp of the harmonic field have yet been performed.

In this Letter, we present a measurement of both the chirp and pulse duration of the fifth harmonic $(15.5 \mathrm{eV})$ of a frequency-doubled Ti:sapphire laser. Our method, illustrated in Fig. 1, is similar to cross-correlation frequencyresolved optical gating techniques which have been used to characterize UV pulses [12,17]. Specifically, we measure the photoelectron signal due to the cross correlation of har-
PACS numbers: $42.65 . \mathrm{Ky}, 32.80 . \mathrm{Rm}$

monics generated by the $400 \mathrm{~nm}$ blue light and an $800 \mathrm{~nm}$ infrared (IR) probe pulse. When the two pulses overlap in time, sidebands appear in the photoelectron spectrum, corresponding to absorption of a harmonic photon together with absorption or emission of one or more IR photons. Since the harmonics are generated by the blue laser light, the sidebands from two consecutive odd harmonics are well separated. This allows us to characterize one harmonic at a time. By delaying one pulse with respect to the other, the duration of the harmonic pulse can be inferred from the delay-dependent intensity of the sidebands $[9,18]$. A chirp in the harmonic frequency makes the location of the sideband also depend on the delay between the pulses, and this dependence can be used to measure the timedependent frequency of the harmonic. The determination of the frequency chirp and pulse duration of the harmonic can thus be done independently. It is also possible to

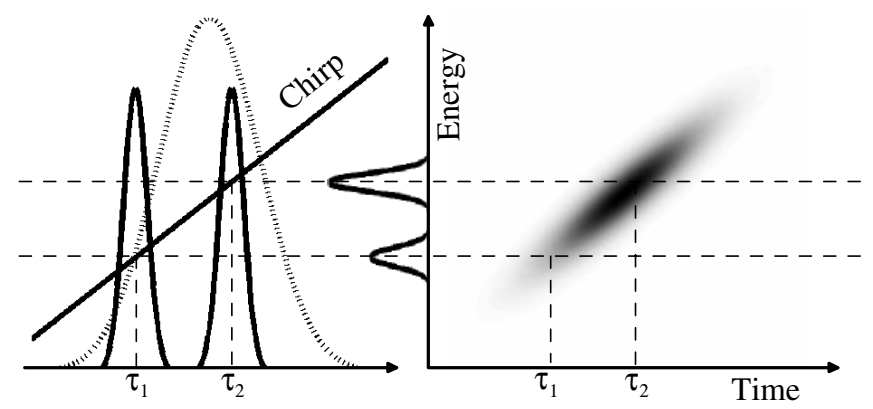

FIG. 1. Principle of the time-frequency characterization of XUV pulses. The dotted curve indicates the XUV pulse, assumed to be linearly chirped, as shown by the straight line. The two short pulses indicated in the solid lines are IR pulses at two different delays relative to the XUV pulse. The position of the electron peak resulting from absorption of an XUV photon and IR photon depends on the time delay. From the measurement of the electron signal and energy as a function of time delay, the pulse duration and chirp of the XUV radiation can be deduced. 
use the delay-dependent width of the photoelectron peak that corresponds to the absorption of one XUV photon to measure pulse durations with femtosecond time resolution $[19,20]$. The application of this method to the measurement of an XUV chirp has been discussed in [19].

In our experimental conditions, we measure that the fifth harmonic exhibits a linear chirp which differs from that predicted by perturbation theory by a small negative amount. In addition, we demonstrate that the chirp of the harmonic can be changed by adding different chirps to the blue driving laser pulse. Comparison of our results to calculations based on the numerical solution of the timedependent Schrödinger equation, together with propagation calculations in the nonlinear medium, suggests that there are several possible origins for the observed timefrequency behavior.

The experimental setup is presented in Fig. 2. Our $1 \mathrm{kHz}$ laser system produces $800 \mathrm{~nm}, 50 \mathrm{fs}$ pulses with an output energy of $\approx 1 \mathrm{~mJ}$. The IR pulses are frequency doubled in a 1.3-mm-long (potassium dihydrogen phosphate) KDP crystal, and the resulting $400 \mathrm{~nm}$ blue light is focused by an $f=200 \mathrm{~mm}$ lens into a $3 \mathrm{~mm}$ windowless gas cell (filled with Xe to a pressure of 1 mbar). The generated odd harmonics are focused by a normalincidence spherical gold mirror into a magnetic-bottle electron spectrometer (MBES), filled with Xe at a static pressure of $10^{-5}$ mbar. The remaining IR light is split off before the harmonic generator by a dichroic mirror and is focused into the MBES. In contrast to previous pulse duration measurements $[9,18-20]$, the harmonic and IR beams are not collinear but cross in the interaction region at an angle of $8^{\circ}$. This does not significantly affect our time resolution, but allows us to manipulate both beams independently. The polarizations of the pump and probe beams are parallel and in the direction of the electron time-of-flight drift tube. The focused intensity of the blue light in the harmonic generation chamber is estimated to

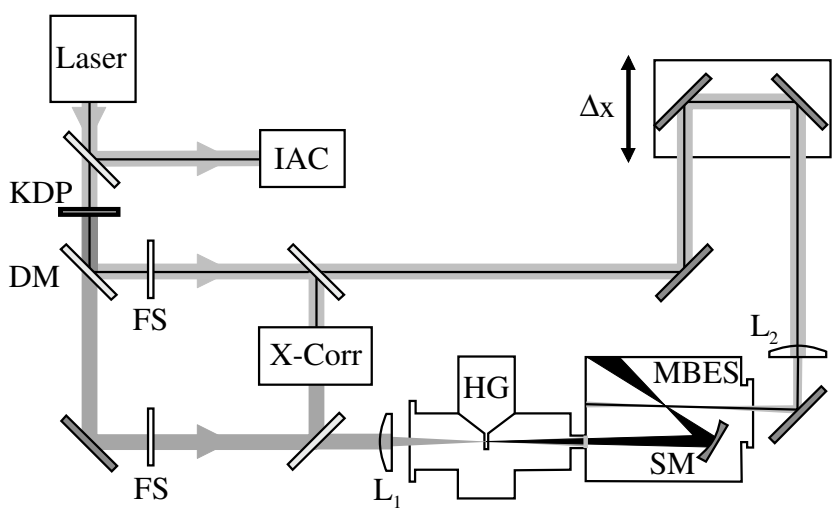

FIG. 2. Experimental setup. The labels are KDP, doubling crystal; DM, dichroic mirror; $\Delta \mathrm{x}$, translation stage; HG, harmonic generator; IAC, interferometric autocorrelator; $\mathrm{L}_{1}, \mathrm{~L}_{2}$, lenses; SM, spherical mirror; MBES, magnetic-bottle electron spectrometer; FS, fused silica plates; X-Corr, cross correlator. be between $0.5 \times 10^{14} \mathrm{~W} / \mathrm{cm}^{2}$ and $2 \times 10^{14} \mathrm{~W} / \mathrm{cm}^{2}$ in the different cases discussed below. The IR intensity in the MBES is approximately $5 \times 10^{12} \mathrm{~W} / \mathrm{cm}^{2}$. Before each series of measurements, we measure the duration of the IR pulse with an interferometric autocorrelator, and that of the blue pulse by cross correlation with the IR (see Fig. 2).

We consider the three configurations indicated in Table I. Both beams propagate through a lens and an entrance window, amounting to $5 \mathrm{~mm}$ of fused silica (FS). The chirp of the blue pulse is varied by adding FS plates into the path of either the IR or the blue beam. In all three cases, the linear dispersion of the IR pulse, induced by propagation through FS and air, is compensated by adjusting the distance between the gratings in the laser compressor. The IR pulse used in the experiment is close to Fourier transform limited, with a pulse duration of $50 \mathrm{fs}$.

The characterization of the chirp of the blue pulse that enters the harmonic generation chamber is performed in the following way: The group delay dispersion (GDD) $\Phi^{\prime \prime}$ of the blue pulse is calculated by adding the GDD resulting from propagation in air and FS, and then subtracting half the GDD induced in the IR pulse. This is valid even for relatively long KDP crystals, where the group velocity mismatch leads to spectral narrowing and pulse broadening [21]. The results are presented in Table I, second column, together with the linear chirp coefficient (column 3)

$$
b=\frac{\Phi^{\prime \prime}}{\tau_{p}^{4}+\Phi^{\prime \prime 2}},
$$

where $\tau_{p}=\Delta \tau_{p} / 2 \sqrt{\ln (2)}$ and $\Delta \tau_{p}$ is the pulse duration (FWHM) for a Fourier transform-limited $400 \mathrm{~nm}$ pulse, which is estimated to be $80 \mathrm{fs}$ from spectrum measurements. In the fourth column we show $b_{H}^{\text {pert }}$, the perturbation theory prediction for the induced chirp on the fifth harmonic, equal to five times that of the driving pulse.

Figure 3 shows a typical photoelectron spectrum. At the bottom, the electron signal is shown as a function of kinetic energy and delay. A lineout is presented at the top of the figure. Electrons due to the absorption of the fifth and seventh harmonics (H5 and H7) give rise to vertical lines, independent of the delay between the pulses. Absorption of a harmonic photon by a xenon atom leads to two lines, separated by the $1.3 \mathrm{eV}$ spin-orbit splitting of the ion core. We observe several sidebands from the ${ }^{2} P_{3 / 2}$ core, as well as a weaker sideband from the ${ }^{2} P_{1 / 2}$ core. We also see a weak line at $5.6 \mathrm{eV}$, which corresponds to the absorption of the fifth harmonic plus a blue photon, leaving the ion in the ${ }^{2} P_{3 / 2}$ core. The broadening observed on the electron peaks at high kinetic energies is due to low spectrometer resolution in this region.

Figure 4 presents the main results of this work. The energy corresponding to absorption of the fifth harmonic plus one IR photon, leaving the ion in the ${ }^{2} P_{3 / 2}$ core, is plotted as a function of the time delay for the three cases 
TABLE I. Spectral and temporal parameters for the three experimental configurations: A $20 \mathrm{~mm}$ FS plate is introduced in the path of the blue beam in (a) and in the path of the IR beam in (c). (b) refers to the configuration where no extra plate was added.

\begin{tabular}{crrccc}
\hline \hline Configuration & $\Phi^{\prime \prime}\left(\mathrm{fs}^{2}\right)$ & $b\left(\mathrm{fs}^{-2}\right)$ & $b_{H}^{\text {pert }}(\mu \mathrm{eV} / \mathrm{fs})$ & $b_{H}^{\exp }(\mu \mathrm{eV} / \mathrm{fs})$ & $\tau_{H}(\mathrm{fs})$ \\
\hline (a) & 2300 & 250 & 810 & 400 & 90 \\
(b) & 400 & 100 & 340 & 15 & 60 \\
(c) & 40 & 10 & 80 & -160 & 70 \\
\hline \hline
\end{tabular}

discussed in Table I. The variation in the sideband photoelectron energy is due to both the chirp of the harmonic (as discussed above) and to the ac Stark shift of the ionization potential caused by the IR laser field. If the IR pulse envelope is symmetric in time, the variation of the sideband energy due to the ac Stark shift, which depends on the IR intensity, is symmetric with respect to time delay, in contrast to the shift caused by the harmonic chirp. Zero delay is found from the maximum of the secondorder sideband. We deconvolute the harmonic chirp from the ac Stark shift effect by fitting the sideband peak to a tilted Gaussian function. This procedure means that we do not have to precisely know the IR intensity. However, only the linear part of the harmonic chirp can be extracted. If the IR pulse duration was much shorter than that of the XUV, the sideband would be generated mostly at time $t_{d}$, where $t_{d}$ is the time delay between the peaks of the harmonic and IR pulses. Since, in our experimental conditions, the IR pulse duration is actually comparable to that of the XUV, the sideband is predominantly generated at the time of

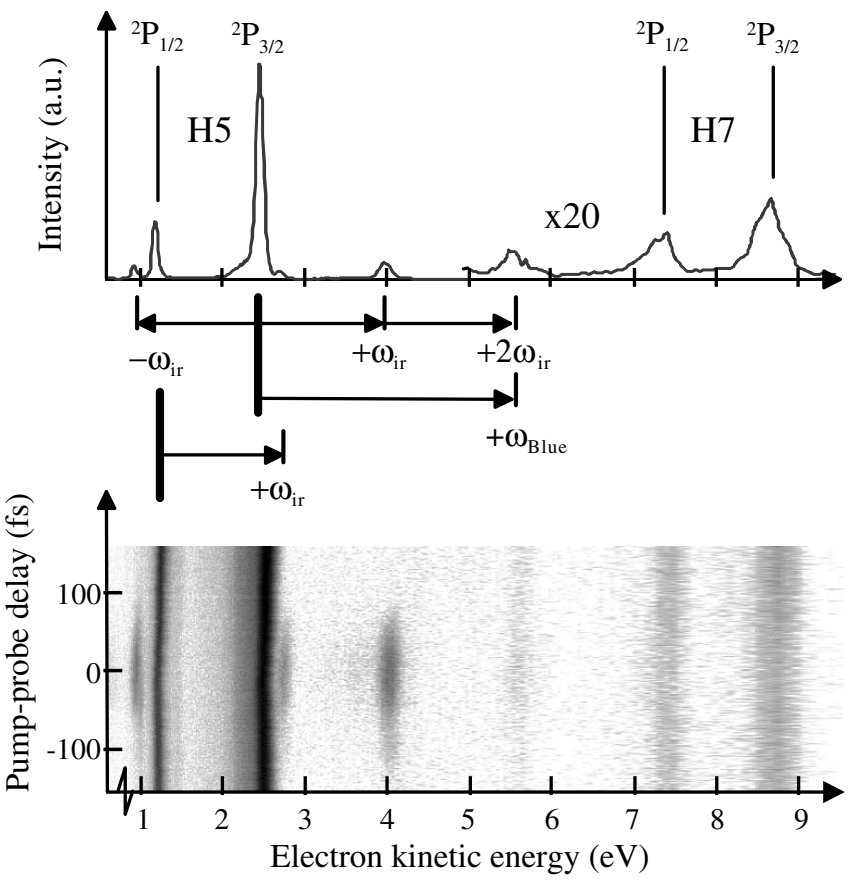

FIG. 3. Bottom: electron signal as a function of kinetic energy and time delay. Top: lineout at zero time delay. The right part is magnified by a factor of 20 . See text for explanation of the different peaks. maximum overlap between the two pulses $t_{\max }<t_{d}$. For Gaussian pulses, $t_{d} / t_{\max }=1+\left(\tau_{\mathrm{IR}} / \tau_{\mathrm{XUV}}\right)^{2} \approx 2$ in our experimental conditions. This factor is accounted for in our determination of the chirp.

The measured linear chirp $b_{H}^{\exp }$ of the fifth harmonic is shown in the fifth column in Table I. The experimental uncertainty is $\approx 40 \mu \mathrm{eV} /$ fs for the first two measurements and $\approx 60 \mu \mathrm{eV} /$ fs for the third measurement, for which the statistics are not as good, because the intensity of the $400 \mathrm{~nm}$ pulse was not as high as in the first two cases. The results demonstrate that when we add material in either the blue or the IR beam we can induce a positive or a negative chirp on the fifth harmonic. From the variation of the sideband amplitude as a function of time delay we also deduce

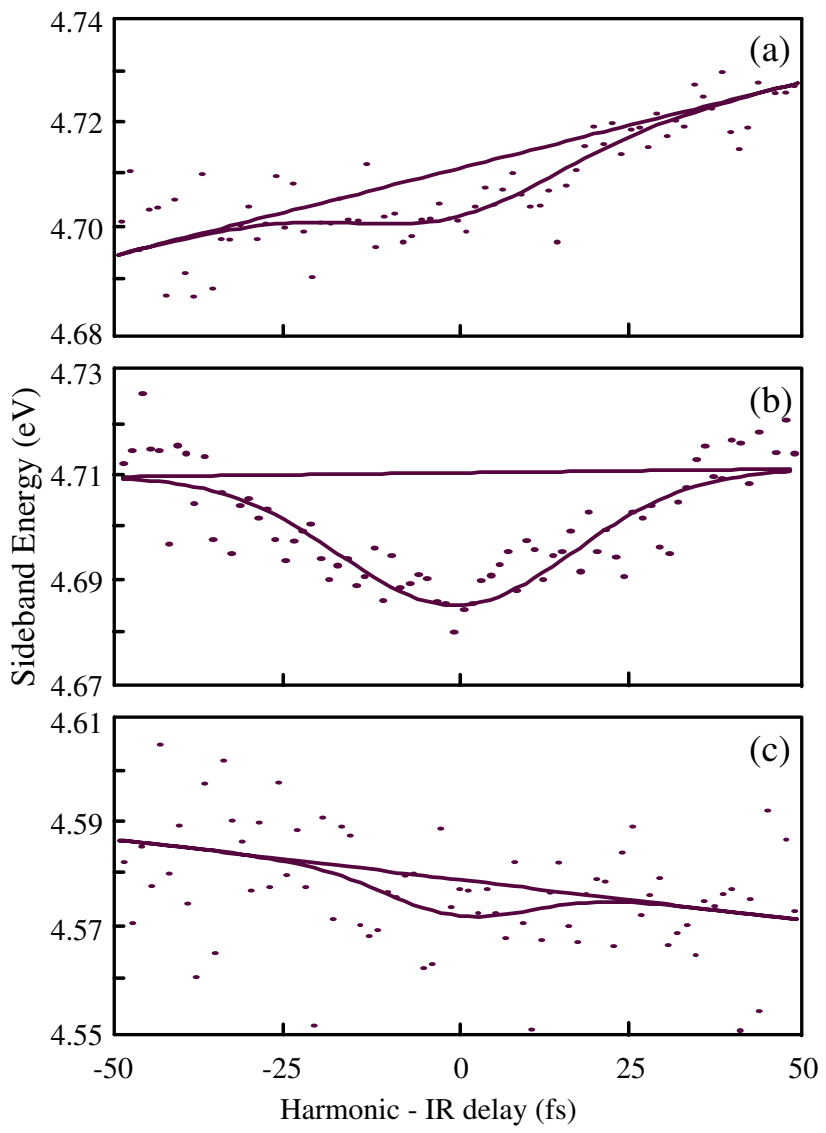

FIG. 4. Energy of the sideband corresponding to absorption of one fifth-harmonic photon plus one IR laser photon, with the ion core left in the $2 P_{3 / 2}$ state, as a function of time delay. (a)-(c) refer to the configurations listed in Table I. 
the pulse duration of the harmonic, shown in the last column in the table. We note that the largest chirp magnitude leads to the longest pulse, and that the (close to) unchirped harmonic has the shortest pulse duration. The chirps listed in column five differ from the perturbative predictions in column four. The quantitative difference between $b_{H}^{\exp }$ and $b_{H}^{\text {pert }}$ varies between -400 and $-200 \mu \mathrm{eV} / \mathrm{fs}$ in the three cases.

To understand the possible origin of these differences, we have performed calculations of both the single atom dipole response and the macroscopic response from a nonlinear medium interacting with a focused laser beam, thereby including effects of propagation and phase matching [22]. We consider intensities close to the estimated saturation intensity for our pulse parameters $\left(5 \times 10^{13} \mathrm{~W} /\right.$ $\mathrm{cm}^{2}$ ). For the fifth harmonic we do not find an intensity dependence of the phase of the dipole moment as discussed in the semiclassical model [23]. There is, however, a time dependence to the single atom phase which can give rise to frequency modulations. We have also performed single atom calculations with chirped driving pulses. We find that at low intensities, or large chirps on the driving pulse, the fifth-harmonic chirp is close to 5 times that of the fundamental. However, at higher intensities and for smaller chirps, comparable to those of this experiment, the perturbative prediction for the single atom harmonic chirp can fail. Macroscopic effects, even in the absence of single atom phase variations, can also give rise to chirps between 100 and $400 \mu \mathrm{eV} / \mathrm{fs}$. The time-dependent depletion of the neutral medium by the intense field, in combination with the geometrical phase of a focused laser beam, gives rise to a chirp which can be positive or negative. For long confocal parameters, we find that this chirp is positive when the focus is after the center of the medium and negative when it is before. For short confocal parameters, however, the chirp can also be negative when the focus is after the medium. Since the confocal parameter is relatively short in this experiment, and the intensity is above saturation in all three cases, it is likely that it is this macroscopic effect that is the origin of the observed harmonic chirp.

In summary, we have measured the pulse duration and linear frequency chirp of the fifth harmonic of $400 \mathrm{~nm}$ radiation, using an energy-resolved cross-correlation method. Our measurement and manipulation of the chirp is the first demonstration of a technique that can be used to characterize the time-frequency behavior of much higher-order harmonics. The detailed study of the influence of experimental conditions on the time-frequency behavior of high harmonics is a prerequisite for the pre- cise control of harmonic properties such as pulse length, spectral shape, and temporal coherence [24].

Support from the Göran Gustafsson Foundation for research in natural sciences and medicine, the Swedish Natural Science Research Council, the Knut and Alice Wallenberg Foundation, and the European Community (FIRE Project ERB-FMGE-CT98-0095 and ATTO Network HPRN-CT-2000-00133), is gratefully acknowledged. M.R. acknowledges the support of the Nordisk Forskerutdanningsakademi (NorFa). K. S. acknowledges the support of the Swedish Foundation for International Cooperation in Research and Higher Education (STINT) and of the National Science Foundation through Grant No. PHY-9733890. U. K. acknowledges the support of the Royal Swedish Academy of Sciences (KVA). Computer time was provided by the National Supercomputer Centre in Sweden.

*Present address: Department of Biophysics, Leiden University, Leiden, The Netherlands.

${ }^{\dagger}$ Present address: Laboratoire de Magnétisme et d'Optique de Versailles (LMOV), CNRS, Versailles, France.

[1] M. Lewenstein et al., Phys. Rev. A 52, 4747 (1995).

[2] M. Bellini et al., Phys. Rev. Lett. 81, 297 (1998).

[3] Z. Chang et al., Phys. Rev. A 58, R30 (1998).

[4] M. B. Gaarde, Opt. Exp. 8, 529 (2001).

[5] I. P. Christov et al., Phys. Rev. Lett. 86, 5458 (2001).

[6] P. Salières et al., Science 292, 902 (2001).

[7] K. J. Schafer et al., Phys. Rev. Lett. 70, 1599 (1993); P. B. Corkum, Phys. Rev. Lett. 71, 1994 (1993).

[8] S. C. Rae et al., Phys. Rev. A 50, 3438 (1994).

[9] T. E. Glover et al., Phys. Rev. Lett. 76, 2468 (1996); Phys. Rev. A 63, 023403 (2001).

[10] C.-G. Wahlström et al., Phys. Rev. A 48, 4709 (1993).

[11] P. M. Paul et al., Science 292, 1689 (2001).

[12] R. Trebino et al., Rev. Sci. Instrum. 68, 3277 (1997).

[13] C. Iaconis and I. A. Walmsley, Opt. Lett. 23, 792 (1998).

[14] T. Sekikawa et al., Phys. Rev. Lett. 83, 2564 (1999).

[15] D. Descamps et al., Phys. Rev. A 64, 031404(R) (2001).

[16] R. Bartels et al., Chem. Phys. 267, 277 (2001).

[17] S. Linden et al., Opt. Lett. 24, 569 (1999).

[18] A. Bouhal et al., Phys. Rev. A 58, 389 (1998).

[19] E. S. Toma et al., Phys. Rev. A 62, 061801 (2000).

[20] M. Drescher et al., Science 292, 1923 (2001); M. Henschel et al., Nature (London) 414, 511 (2001).

[21] E. Sidick et al., J. Opt. Soc. Am. B 12, 1713 (1995).

[22] A. L'Huillier et al., Phys. Rev. A 46, 2778 (1992).

[23] We note that such an intensity-dependent phase would be slowly changing with intensity at $\lambda=400 \mathrm{~nm}$, since the phase scales as $\lambda^{3}$.

[24] L. Roos et al., J. Phys. B 34, 5041 (2001). 